\title{
Cardiac magnetic resonance in pericardial disease
}

\author{
Giovanni D Aquaro ${ }^{1 *}$, Andrea Barison ${ }^{1}$, Giancarlo Todiere ${ }^{1}$, Alessandro Cagnolo', Michele Emdin ${ }^{1}$, \\ Massimo lombardi ${ }^{2}$ \\ From 18th Annual SCMR Scientific Sessions \\ Nice, France. 4-7 February 2015
}

\section{Background}

Cardiac magnetic resonance (CMR) allows the detection of acute inflammation, fusion and thickening of pericardial layers and of effusion. We sought to evaluate the diagnostic and prognostic value of CMR in the setting of pericardial disease.

\section{Methods}

CMR was performed in 146 patients (85 males, aged 56 \pm 16 years) with clinical suspicion of acute pericarditis (AP, $n=46$ ), pericardial effusion without inflammation $(\mathrm{PE}, \mathrm{n}=47)$, and constrictive pericarditis $(\mathrm{CP}, \mathrm{n}=53)$. Final diagnosis was ascertained by a combined evaluation of different CMR sequences. Patients were followed-up for all-cause and cardiac death.

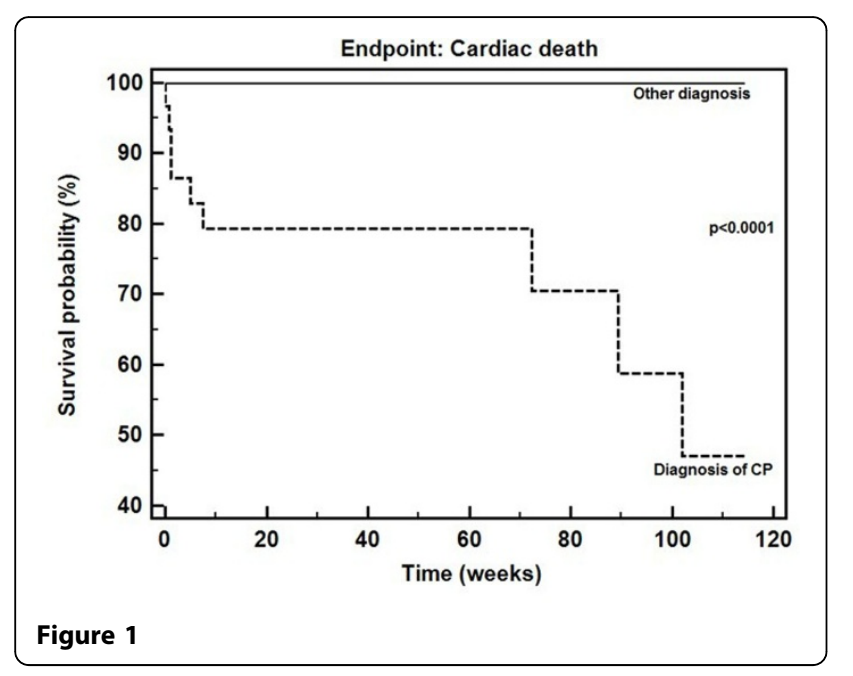

${ }^{1}$ Fondazione Toscana G.Monasterio, Pisa, Italy

\section{Results}

The final diagnosis was AP in 56 patients (38\%), PE in $38(26 \%)$, and CP in $42(29 \%)$. Pericardial disease was excluded in 7 patients (5\%), with CMR evidence of abundant epicardial fat. The initial diagnosis was changed by CMR in $21 \%$ of patients. Initial suspicion was confirmed in 40/46(87\%) patients with AP suspicion (alternative CP diagnosis: 2, PE: 1; no pericardial disease: 3). PE was confirmed by CMR in 35/47(75\%) patients (10 with a final AP diagnosis, 2 patients with absent pericardial disease). A CP diagnosis was confirmed in 40/53(75\%), while 6 presented signs of AP (resembling a "transient constrictive pericarditis"), 5 PE (as "effusiveconstrictive pericarditis"), and 2 showed no pericardial disease. At Kaplan-Meyer analysis, on a 551-day median follow-up (IQ range 330-1110), patients diagnosed with $\mathrm{CP}$ had a worse prognosis than AP or PE patients ( $\mathrm{P}$ $<0.001)$.

\section{Conclusions}

CMR has an additive value, as compared to conventional clinical evaluation, both for differential diagnosis of pericardial disease and for risk stratification.

\section{Funding}

N/A.

\section{Authors' details}

${ }^{1}$ Fondazione Toscana G.Monasterio, Pisa, Italy. ${ }^{2}$ Multimodality Cardiac Imaging Section, San Donato, Milano, Italy.

Published: 3 February 2015

doi:10.1186/1532-429X-17-S1-P377

Cite this article as: Aquaro et al:: Cardiac magnetic resonance in pericardial disease. Journal of Cardiovascular Magnetic Resonance 201517 (Suppl 1):P377.

\section{() Biomed Central}

